\title{
Minimally invasive transforaminal lumbar interbody fusions and fluoroscopy: a low-dose protocol to minimize ionizing radiation
}

\author{
Justin C. Clark, M.D., ${ }^{1}$ Gary Jasmer, R.T.(R), ${ }^{2}$ Frederick F. Marciano, M.D., Ph.D., $, 1,3$ \\ and Luis M. Tumialán, M.D. ${ }^{1,3}$
}

${ }^{1}$ Division of Neurological Surgery, Barrow Neurological Institute, St. Joseph's Hospital and Medical Center, Phoenix; and Divisions of ${ }^{2}$ Radiology and ${ }^{3}$ Neurological Surgery, Scottsdale Healthcare System, Scottsdale, Arizona

\begin{abstract}
Object. There is an increasing awareness of radiation exposure to surgeons and the lifelong implications of such exposure. One of the main criticisms of minimally invasive transforaminal lumbar interbody fusion (MIS TLIF) is the amount of ionizing radiation required to perform the procedure. The goal in this study was to develop a protocol that would minimize the fluoroscopy time and radiation exposure needed to perform an MIS TLIF without compromising visualization of the anatomy or efficiency of the procedure.

Methods. A retrospective review of a prospectively collected database was performed to review the development of a low-dose protocol for MIS TLIFs in which a combination of low-dose pulsed fluoroscopy and digital spot images was used. Total fluoroscopy time and radiation dose were reviewed for 50 patients who underwent single-level MIS TLIFs.

Results. Fifty patients underwent single-level MIS TLIFs, resulting in the placement of 200 pedicle screws and 57 interbody spacers. There were 28 women and 22 men with an average age of 58.3 years (range $32-78$ years). The mean body mass index was $26.2 \mathrm{~kg} / \mathrm{m}^{2}$ (range $17.1-37.6 \mathrm{~kg} / \mathrm{m}^{2}$ ). Indications for surgery included spondylolisthesis (32 patients), degenerative disc disease with radiculopathy (12 patients), and recurrent disc herniation (6 patients). Operative levels included 7 at L3-4, 40 at L4-5, and 3 at L5-S1. The mean operative time was 177 minutes (range 139-241 minutes). The mean fluoroscopic time was 18.72 seconds (range 7-29 seconds). The mean radiation dose was $0.247 \mathrm{mGy} * \mathrm{~m}^{2}$ (range $0.06046-0.84054 \mathrm{mGy}^{*} \mathrm{~m}^{2}$ ). No revision surgery was required for any of the patients in this series.

Conclusions. Altering the fluoroscopic technique to low-dose pulse images or digital spot images can dramatically decrease fluoroscopy times and radiation doses in patients undergoing MIS TLIFs, without compromising image quality, accuracy of pedicle screw placement, or efficiency of the procedure.
\end{abstract}

(http://thejns.org/doi/abs/10.3171/2013.5.FOCUS13144)

KEY WoRDS $\bullet$ radiation exposure $\bullet$ radiation safety $\bullet$ fluoroscopy
minimally invasive surgery $\bullet$ transforaminal lumbar interbody fusion $\bullet$
spine surgery $\bullet \quad$ lumbar spine

I

$\mathrm{N}$ recent years, an understandable concern has arisen in the literature regarding the amount of ionizing radiation exposure to a surgeon during minimally invasive spinal fusions. ${ }^{20,23,25,30,35}$ Techniques for MIS have

\footnotetext{
Abbreviations used in this paper: $\mathrm{ABC}=$ automatic brightness control; AEC $=$ automatic exposure control; ALARA $=$ as low as reasonably achievable; $\mathrm{AP}=$ anteroposterior; $\mathrm{BMI}=$ body mass index; $\mathrm{kVp}=$ kilovolt potential; MIS = minimally invasive surgery; ODI = Oswestry Disability Index; SF-36 = 36-Item Short Form Health Survey; TLIF = transforaminal lumbar interbody fusion; VAS $=$ visual analog scale.
}

traditionally required higher doses of ionizing radiation than open procedures, a fact once held to be an unavoidable aspect of MIS techniques..$^{22,25,32,33}$ This increased awareness has led to surgeons exploring technologies such as intraoperative navigation, and may be a factor in surgeons' reluctance to incorporate minimally invasive techniques into their practices..$^{15,18,29,31,35}$ At the same time, there has been a trend in the recent literature that examines techniques for lowering exposure to ionizing radiation in surgeons and patients undergoing fluoroscopically guided procedures in the fields of interventional cardiology, urology, and pediatrics..$^{2,5-9,11,21,28}$ As minimally in- 
vasive techniques continue to mature, part of this maturation process will entail further addressing the issue of exposure to ionizing radiation.

It is this increased awareness of radiation exposure and concern for the lifelong implications of total radiation dose that was the impetus behind our reevaluation of their fluoroscopic technique for minimally invasive lumbar fusions. We sought to modify the technique to minimize the radiation dose and fluoroscopy times without compromising the accuracy of pedicle screw and interbody placement or the efficiency of the operation. In this report, we describe a low-dose fluoroscopy protocol that was developed and used in 50 patients undergoing a single-level MIS TLIF. Throughout these 50 cases, we abandoned conventional (continuous interlaced) fluoroscopy and explored the use of digital spot imaging and low-dose pulsed fluoroscopy to refine a low-dose protocol for the MIS TLIF. The fluoroscopy times and radiation dose were prospectively collected and then retrospectively reviewed once the protocol was refined. The times and exposures from the low-dose protocol were then compared with the experience in the recent literature.

We surmise that modification of the fluoroscopic technique may dramatically decrease the amount of fluoroscopy needed for MIS TLIFs, and over time will significantly impact the surgeon's lifetime dose of radiation exposure.

\section{Methods}

A low-dose protocol was developed modifying the standard settings of the fluoroscopy unit to a lower dose of radiation and shorter acquisition time per image obtained. A combination of 2 settings was used: digital spot or pulsed low-dose fluoroscopy. The authors used either a General Electric OEC 9900 C-arm system or General Electric OEC 9900 Super C series systems for all cases. The settings on the fluoroscopic unit were changed from the standard settings to the following: "orthopedic exam profile" and "digital spot" or "low dose" and "pulse." For the digital spot protocol, a manual technique was implemented adjusting the $\mathrm{kVp}$ and the $\mathrm{mA}$. Each of these settings was adjusted to optimize image quality and minimize radiation dose. Modification of the $\mathrm{kVp}$ and $\mathrm{mA}$ was based on the BMI of the patient, with greater values for larger patients to achieve adequate penetration and thereby an acceptable image. (Both $\mathrm{kVp}$ and $\mathrm{mA}$ will be discussed in greater detail in the Discussion section.) For the low-dose pulsed fluoroscopy protocol, no modification of the $\mathrm{kVp}$ and $\mathrm{mA}$ was needed because the $\mathrm{ABC}$ and AEC adjusts the $\mathrm{kVp}$ and the $\mathrm{mA}$.

Once an image is acquired for either pulsed fluoroscopy or digital spot, manual adjustment of the contrast and brightness by the fluoroscopic technician allows for clear visualization of the bony anatomy. All subsequent images obtained are digital spot images or pulsed lowdose fluoroscopy. Each of these techniques has an acquisition time of approximately 0.15 seconds, compared with conventional fluoroscopy, which has a minimal acquisition time of 1 second. Throughout development of this low-dose protocol, all MIS TLIFs performed by the se- nior author (L.M.T.) between July 2010 and March 2013 were recorded in a prospective database approved by the institutional review board of the Scottsdale Healthcare System. Patient demographic data, indications for surgery, level, operating time, blood loss, fluoroscopy time, and total radiation dose were prospectively collected.

Patients with the diagnosis of spondylolisthesis, degenerative disc disease, and recurrent disc herniation who had failed 3-6 months of nonoperative measures and who had been selected as candidates for MIS TLIFs were included in this study. Preoperative and postoperative ODI, SF-36, and VAS back and leg scores were collected. After patients were positioned for surgery, the fluoroscope was brought into the field and localization images with a spinal needle on the appropriate level were obtained implementing the digital spot. Because these are the first images obtained, the fluoroscopic technician manually adjusts the $\mathrm{kVp}$ and $\mathrm{mA}$ as well as the contrast and brightness setting to optimize subsequent images while minimizing the radiation dose. Two $28-\mathrm{mm}$ incisions were planned 3-3.5 $\mathrm{cm}$ off of midline, and additional fluoroscopic images (either digital spot or pulsed low dose) were obtained for docking the retractors onto the facets to be operated on.

After bilateral exposure of the pars interarticularis, transverse process, and facet joints, lateral fluoroscopic digital spot images were obtained to confirm pedicle screw entry points, trajectories, and to establish screw length. On average, 4 to 7 digital spot images would be obtained per pedicle, and all 4 pedicles screws would be placed with a range of 16-28 fluoroscopic digital spot images. As the pedicles were accessed, the Lenke pedicle probe, pedicle taps, and pedicle screws were each stimulated to $20 \mathrm{~mA}$ to ensure that a compound motor action potential was not activated at the relevant nerve root on free-running electromyography. Any stimulation of a nerve root prompted an AP image and reevaluation of the pedicle screw entry point. In the absence of stimulation, the integrity of the pedicle was further ensured with a ball-tipped probe. Once all 4 pedicle screws were placed, a provisional rod was secured on the side contralateral to the transforaminal approach, the fluoroscope was transitioned to the head of the bed, and the operating microscope brought into the field.

A laminectomy and complete facetectomy were then performed on the symptomatic side, the thecal sac and the exiting and traversing nerve roots were decompressed, and the disc space accessed and prepared. Restoration of the disc height was achieved using paddle distractors with the contralateral rod capturing the height. On completion of the discectomy, the operating microscope was removed and the fluoroscope repositioned for placement of the interbody spacer.

The appropriate interbody trial spacer was then secured into the interbody space. Additional digital spot or pulsed low-dose fluoroscopic images were obtained to determine the ideal size of the interbody spacer (3-5 images). Once the appropriate size was selected, 1 or 2 interbody spacers (Crescent; Medtronic Sofamor-Danek) were rotated into position within the disc space with digital spot or pulsed low-dose fluoroscopic images. An additional 5-7 images were obtained for interbody placement. 
After satisfactory placement of the interbody, 1 final AP digital spot image was obtained to confirm midline position of the spacer(s) and convergence of the pedicle screws.

At the end of the procedure, total fluoroscopy time, radiation dose, blood loss, and operating time were collected and recorded in the database. All patients underwent postoperative standing AP and lateral radiographs to evaluate instrumentation and restoration of disc height either the afternoon of surgery or on the 1st postoperative day. All patients were followed for a minimum of 6 months, with clinical evaluations at 1 month, 3 months, 6 months, and 1 year (when possible). Anteroposterior and lateral radiographs were obtained at each visit, with flexion-extension studies obtained at 3 and 6 months, along with ODI, SF-36, and VAS back and leg scores. After refinement of the low-dose protocol with the combination of digital spot and low-dose pulsed fluoroscopy, the authors reviewed the quality of images obtained, radiation dose, and fluoroscopy times for the last 50 surgically treated cases.

\section{Results}

Fifty patients underwent single-level MIS TLIF procedures, resulting in the placement of 200 pedicle screws and 57 interbody spacers (43 patients had 1 interbody spacer, and 7 patients underwent placement of 2 interbodies). There were 28 women and 22 men with an average age of 58.3 years (range 32-78 years). The mean BMI was $26.2 \mathrm{~kg} / \mathrm{m}^{2}$ (range $17.1-37.6 \mathrm{~kg} / \mathrm{m}^{2}$; Table 1). Indications for surgery included spondylolisthesis (32 patients), degenerative disc disease with radiculopathy (12 patients), and recurrent disc herniation (6 patients). Operative levels included 7 at L3-4, 40 at L4-5, and 3 at L5-S1 (Table 2). The mean operating time was 177 minutes (range 139-241 minutes). The mean fluoroscopic time was 18.72 seconds (range 7-29 seconds). The mean radiation dose was $0.247 \mathrm{mGy}^{*} \mathrm{~m}^{2}$ (range 0.06046-0.84054 $\mathrm{mGy}^{*} \mathrm{~m}^{2}$; Table 3). Retrospective review of the fluoroscopic images demonstrated greater detail of the bony anatomy on the digital spot images at higher $\mathrm{mA}$ than on low-dose pulsed fluoroscopy.

Probing and stimulating the pedicle resulted in the activation of a compound motor action potential identified on free-running electromyography on 4 occasions in 4 separate cases. In each case an AP digital spot image was obtained at the time of the stimulation. In 3 cases the entry point was found to be too medial and in 1 case it was found to be too lateral. In all 4 occasions, the pedicle

TABLE 1: Demographic data in 50 patients who underwent MIS TLIF

\begin{tabular}{ll}
\hline \multicolumn{1}{c}{ Characteristic } & \multicolumn{1}{c}{ Value } \\
\hline mean patient age in yrs (range) & $58.3(32-78)$ \\
no. of men (\%) & $22(44)$ \\
no. of women $(\%)$ & $28(56)$ \\
mean BMl in $\mathrm{kg} / \mathrm{m}^{2}$ (range) & $26.2(17.1-37.6)$ \\
\hline
\end{tabular}

TABLE 2: Surgical data in 50 patients who underwent MIS TLIF

\begin{tabular}{cc}
\hline Lumbar Level & No. of Patients \\
\hline L3-4 & 7 \\
L4-5 & 40 \\
L5-S1 & 3 \\
\hline
\end{tabular}

screw entry point was adjusted and the pedicle was successfully instrumented without subsequent irritation of a nerve root at $20 \mathrm{~mA}$. We had $100 \%$ follow-up at 1 month and $96 \%$ follow-up at 3 months. Six-month follow-up has not been completed for all the patients in the series at this time. The mean follow-up at this time is 5.4 months (range 3-12 months). The mean preoperative ODI, SF36, and VAS (back and leg) scores were as follows: 42.8, $20.9,7.2$, and 7.8, respectively. At 3 months, ODI was 17.2, SF-36 was 58.4, and VAS back and leg were 4.2 and 1.8 , respectively. In the postoperative course, there were no symptomatic breaches or revisions required among the 200 pedicle screws placed. There was 1 incidental durotomy that was primarily repaired at the time of surgery. There were no postoperative infections in the patients in this series.

\section{Discussion}

The International Commission on Radiological Protection has introduced the principle of ALARA as a system for limiting the dose of ionizing radiation received by individuals. ${ }^{37}$ There are 3 elements to this principle that may be directly applied to fluoroscopy in the MIS TLIF: justification, dose limitation, and optimization. ${ }^{3,12,13}$ It is the application of these 3 elements of the ALARA principle in the context of an MIS TLIF that is the main focus of this current work.

When applied to the MIS TLIF, justification would mean that the proposed fluoroscopic image that will cause radiation exposure to the surgeon and the operating room team will yield actionable information such as adjusting an entry point for a pedicle screw, altering a trajectory, or adjusting an interbody spacer. Throughout the development of this protocol, we attempted to limit acquiring images if it did not affect screw placement, trajectory, or interbody position. We also attempted to identify simultaneously 2 pedicle screw entry points with 1 image, that is, the first surgeon working at the caudal pedicle and the second surgeon working at the rostral pedicle. Dose limitation entails setting annual dose limits for surgeons and operating room personnel, which under ideal circumstances would be a fraction of the actual dose limit. The

TABLE 3: Time of surgery and radiation data in $\mathbf{5 0}$ patients who underwent MIS TLIF

\begin{tabular}{lc}
\hline \multicolumn{1}{c}{ Factor } & Mean Value (range) \\
\hline op time in min & $177(139-241)$ \\
fluoroscopic duration/case in sec & $18.72(7-29)$ \\
radiation dose in $\mathrm{mGy}^{*} \mathrm{~m}^{2}$ & $0.247(0.061-0.841)$ \\
\hline
\end{tabular}


National Council for Radiation Protection has set these limits, allowing annual exposure for radiation workers to 5 rem to the body and 50 rem to an extremity. We have set an annual dose limit of one-tenth of this value.

Finally, optimization in this context entails modifying the fluoroscopic technique in a manner that will optimize the visualization of the requisite anatomy for safe instrumentation of the spine while minimizing the radiation. It is this element of the ALARA principle where we believe the greatest impact can be made in the MIS TLIF. To implement optimization of the fluoroscopic technique, a greater understanding of fluoroscopy and the basics of radiation physics is needed, specifically the 3 variables in fluoroscopy that produce the ionizing radiation: $\mathrm{kVp}, \mathrm{mA}$, and acquisition time.

Fluoroscopy produces ionizing radiation by an electrically heated filament (cathode) within the fluoroscopy tube that is directed to the target (anode). The cathode generates electrons that are accelerated from the filament to the tungsten target by the application of voltage to the tube. The energy gained by the electron is the potential difference between the anode and the cathode and may be expressed as the $\mathrm{kVp}$. The quantity of electron flow or current in the x-ray tube is described in units of $\mathrm{mA}$. The rate of ionizing radiation produced is directly proportional to the current or $\mathrm{mA}$. Therefore the higher the $\mathrm{mA}$, the more electrons are striking the tungsten target, thereby producing more $\mathrm{x}$-rays. The voltage (that is, $\mathrm{kVp}$ ), on the other hand, determines the maximum x-ray energy produced. ${ }^{24}$ Understanding these values allows for their modification in the use of fluoroscopy, specifically a digital spot image and pulsed fluoroscopy.

There are 2 general types of fluoroscopy: continuous interlaced (conventional) and pulsed fluoroscopy. It has been demonstrated in the literature that the use of pulsed fluoroscopy may reduce fluoroscopy time by $76 \%$ and radiation dose by $64 \%$ compared with continuous fluoroscopy. ${ }^{11,14,19,27,28}$ The higher radiation dose and fluoroscopy time are due to the acquisition time on 1 image and the automated settings of $\mathrm{kVp}$ and $\mathrm{mA}$. Conventional fluoroscopy is typically performed using 2-5 mA of current with a peak electrical potential of $90-125 \mathrm{kVp}$. The central processing unit of the fluoroscopic unit interlaces a single frame or image every 0.03 seconds. An ABC and AEC in most fluoroscopic units adjusts these values to achieve an optimal image. This may come with the consequence of increased ionizing radiation and acquisition time. ${ }^{26}$ Pulsed fluoroscopy, as the name would suggest, pulses the $\mathrm{x}$-ray beam acquiring an image during a brief pulse of as little as 0.10 seconds. Therefore, the radiation dose is decreased by reducing the time that the x-ray beam is on. The low-dose setting further lowers the amount of ionizing radiation; however, this results in a lower-quality image because the eye averages fewer independent frames. In essence, a low-dose pulsed fluoroscopic image represents a compromise between dose and image quality.

An alternative to low-dose pulsed fluoroscopy is a digitally acquired image also known as a digital spot image. Although digitally recorded images have better contrast resolution than pulsed fluoroscopy images, they suffer from lower spatial resolution. A digital spot has the added benefit (and liability) of manually adjusting the $\mathrm{kVp}$ and $\mathrm{mA}$, which may further lower the radiation dose. Such a technique may achieve levels as low as $0.7 \mathrm{~mA}$ and $80 \mathrm{kVp}$ without compromising the quality of the image. The main disadvantage of the digital spot is the need for a dedicated fluoroscopic technician at the ready to adjust the values of $\mathrm{kVp}, \mathrm{mA}$, brightness, and contrast to optimize the images. Hence, this is a more labor-intensive technique. Over the course of the 50 cases reported in this series, the authors tended to favor the digital spot image when greater image quality was needed; that is, for pedicle screw entry points. The superior quality of image achieved compared with the pulsed fluoroscopic image at the same acquisition time outweighs the slight increase in radiation dose (Fig. 1). However, pulsed fluoroscopy is adequate for setting pedicle screw trajectories and placement of the interbody at a lower dose of radiation (Fig. 2). For finer detail, specifically when determining the medial boundary of the pedicle on an AP view, digital spot is preferable (Fig. 3).

The third variable that may have an impact on radiation dose is the acquisition time of an image. With standard settings, the shortest acquisition time achieved using conventional fluoroscopy is 1 second. Therefore, the number of images obtained will correlate with the fluoroscopy time. If one assumes 5 images to dock the MIS retractors, 5 images per pedicle, 5 images for preparing the disc space and placing the interbody trial spacer, and 5 images for placement of the permanent interbody spacer, the minimum one could achieve with standard fluoroscopic settings is 35 seconds, which is still well below the reported mean in the literature of approximately 86 seconds. The difference between these 2 numbers may be attributable to the actual mechanics of obtaining a fluoroscopic image. The length of this activity is contingent on 2 separate entities. The first is the fluoroscopic technician's deftness in activating and releasing the fluoroscopy switch for the shortest duration possible. The second is how rapidly the $\mathrm{ABC}$ and $\mathrm{AEC}$ achieves optimal exposure for the various factors ( $\mathrm{kVp}$ and $\mathrm{mA}$ ) to provide adequate spatial and contrast resolution of the image. Collectively in practice, this results in each acquisition requiring approximately 1 second of fluoroscopy, but it may be as long as 2 or 3 seconds each.

Both pulsed fluoroscopy and digital spot lower the acquisition times and thereby radiation dose. In the scenario detailed above, such a setting would decrease fluoroscopy time from 35 seconds to 8 seconds. As a proof of principle, the lowest fluoroscopy time with the low-dose digital in our series was 7 seconds. In our opinion the advantage to the digital spot image is that it affords the best spatial and contrast resolution and overall optimum image detail with the shortest acquisition time at radiation doses that may be manually adjusted.

Thus, modifying a technique that minimizes acquisition time and manually setting the $\mathrm{kVp}$ and $\mathrm{mA}$ with a digital spot achieves the ALARA goal of optimization. It is the application of this technique that allowed us to achieve mean fluoroscopy time of 18.72 seconds, with times as low as 7 seconds. We initially used a combination of digital spot and low-dose pulsed fluoroscopy to 

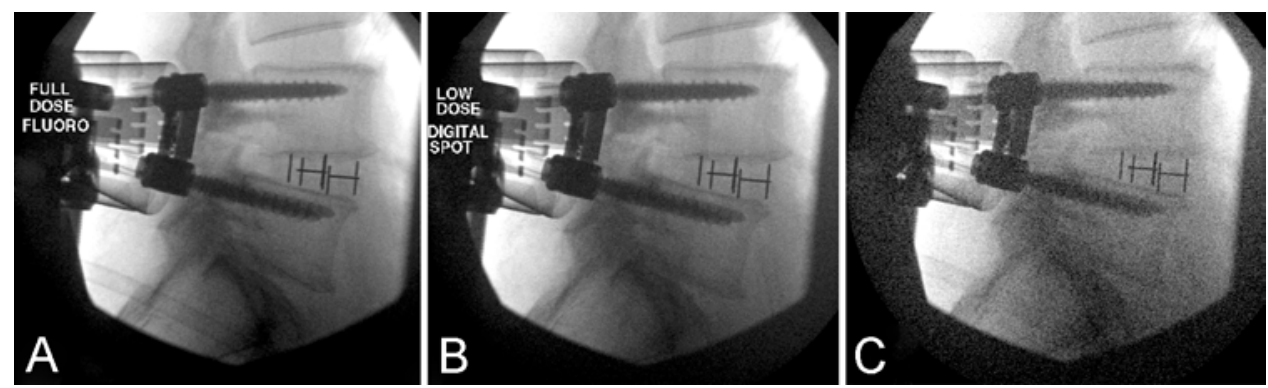

FIG. 1. Lateral fluoroscopic images obtained using conventional fluoroscopy (A), low-dose digital spot (B), and pulsed lowdose fluoroscopy $(\mathbf{C})$ in a patient undergoing an MIS TLIF. The full-dose fluoroscopic image was obtained using $118 \mathrm{kVp}$ and 5.48 $\mathrm{mA}$ with an acquisition time of 1 second, whereas the low-dose digital spot required $120 \mathrm{kVp}$ and increased current of $21.60 \mathrm{~mA}$; however, the acquisition time is 0.15 seconds. Hence, over the span of an entire procedure, the net dose is less than conventional fluoroscopy. The increased current in the digital spot makes these images almost indistinguishable. Finally, the pulsed low-dose fluoroscopy has the lowest current at $2.44 \mathrm{~mA}$, the lowest potential difference at $114 \mathrm{kVp}$, with an acquisition time of 0.15 seconds; however, this comes at the expense of the quality of the image.

achieve lower doses and shorter fluoroscopic times. Over the course of the 50 cases reported herein, the trend has been to favor the digital spot image acquisition. This technique allows for 7 distinct images to be obtained over the span of 1 second, with a higher-quality image than may be acquired with pulsed fluoroscopy and significantly less radiation than conventional fluoroscopy. As mentioned above, the main disadvantage to the digital spot technique is the need for a dedicated fluoroscopic technician capable of adjusting the settings to minimize radiation and optimize the image quality.

Among the operating room personnel, it is not surprising that the surgeon receives higher mean radiation doses by being positioned next to the patient, and every effort should be made to minimize that exposure. ${ }^{23}$ The literature has demonstrated that MIS TLIFs have greater fluoroscopic requirements than comparable open TLIF procedures..$^{25,32,33}$ Bindal et al. ${ }^{4}$ reported an average fluoroscopic time of 1.69 minutes per case with an average of 1.38 levels treated per patient with MIS TLIF techniques, resulting in 73.5 seconds per level. Wang and col-
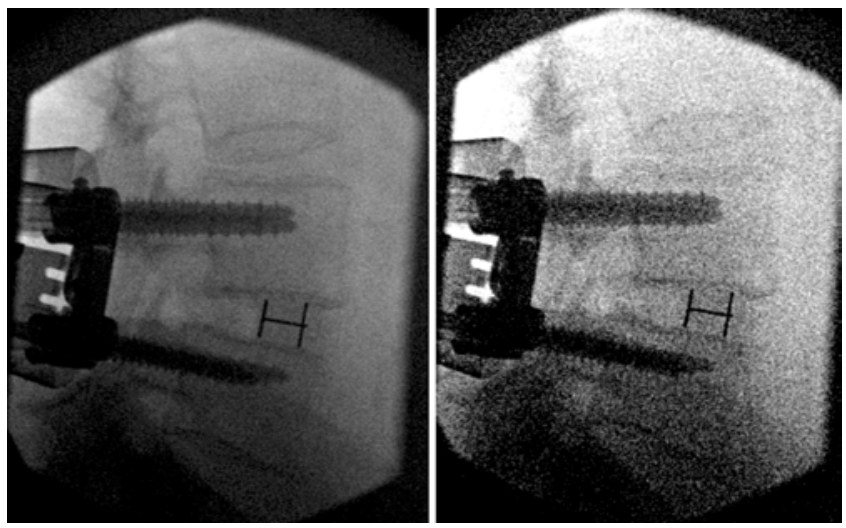

FIG. 2. Lateral fluoroscopic images obtained in a morbidly obese patient (BMI $33.8 \mathrm{~kg} / \mathrm{m}^{2}$ ) undergoing an L4-5 TLIF with low-dose digital spot (left) and low-dose pulsed fluoroscopy (right). Whereas digital spot imaging provides greater detail, the low-dose pulsed fluoroscopy provides an adequate image for interbody spacer placement and pedicle screw trajectory at a lower dose of radiation. Confirming pedicle screw entry points may require finer detail provided by the digital spot at the same acquisition time but a higher radiation dose. leagues ${ }^{33}$ used fluoroscopy for percutaneous placement of 168 pedicle screws in 42 patients, requiring an average of 84 seconds of exposure time. In a later study, Wang and colleagues ${ }^{34}$ also reported that during revision surgery, MIS TLIF fluoroscopy requirements for 25 patients undergoing 28 levels of operation were 73 seconds, or approximately 65 seconds per single-level case. In another work, Wang and colleagues ${ }^{32}$ reported 46 seconds of fluoroscopic time for their single-level MIS TLIF in obese patients. Villavicencio et al. ${ }^{31}$ reported using 133 seconds of fluoroscopy for image-guided single-level MIS TLIF. Kim and colleagues reported decreased fluoroscopy usage in single-level unilateral MIS TLIF cases for intraoperative navigation performed using Iso-C that required 57.1 seconds, compared with standard 2D fluoroscopy that required 147.2 seconds. ${ }^{18}$

By comparison, in the current work we report an average of 18.72 seconds of fluoroscopy by implementing a low-dose technique. This represents an $80 \%$ reduction in fluoroscopy time and is actually less than the reported times for open lumbar fusion cases. It is important to point out that it is not the surgical technique but the fluoroscopic technique that accounts for this large variation between the mean fluoroscopy times in the literature compared with the times reported herein. In all likelihood, a low-dose fluoroscopic technique applied to any of the series listed above would have yielded similar results. To demonstrate this point, the final column of Table 4 assumes an acquisition time of 0.15 seconds for the reported series. When multiplied by the fluoroscopy time, the values generated fall within the range of times seen in our series. It is likely that the number of images obtained for the cases in the current series and those in the reported literature are comparable in number.

With the refinement of the current low-dose protocol, further study is needed to evaluate the implementation of this technique on a prospective basis. To that end, we have begun a prospective study to further examine radiation dose and the impact on fluoroscopy times. It will be equally important to evaluate the application of low-dose protocols in multiple centers.

The impetus behind minimizing radiation for MIS TLIFs and all spine cases, minimally invasive or open, is the recent increased awareness of radiation exposure 

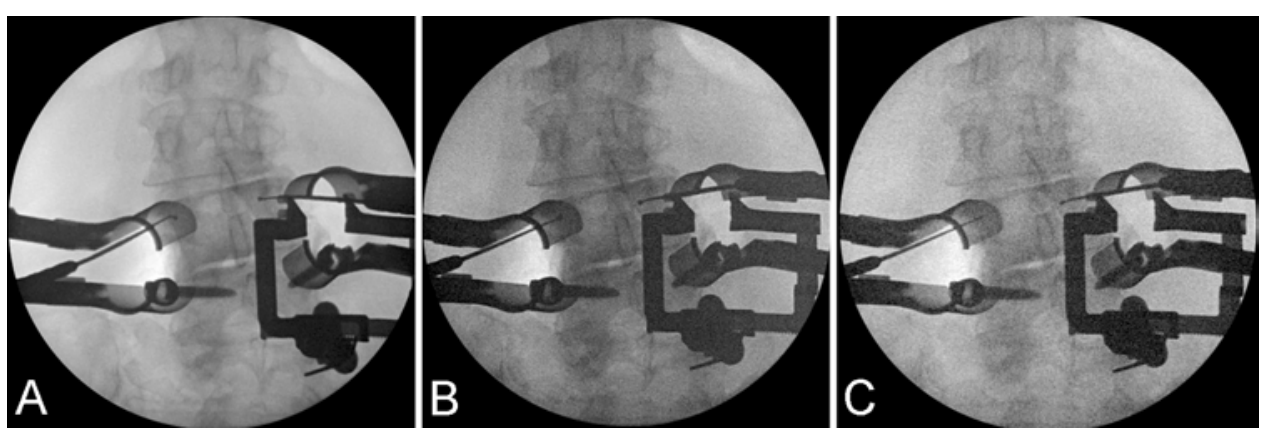

FIG. 3. Anteroposterior fluoroscopic images of a patient undergoing an L3-4 TLIF. A: Conventional fluoroscopy obtained with $93 \mathrm{kVp}, 4.1 \mathrm{~mA}$, and acquisition time of 1 second. B: Low-dose digital spot obtained with $82 \mathrm{kVp}, 0.85 \mathrm{~mA}$, and an acquisition time of 0.15 seconds. C: Low-dose pulsed fluoroscopy obtained with $97 \mathrm{kVp}, 1.4 \mathrm{~mA}$, and acquisition time of 0.15 seconds. Detail of the pedicle is lost with the low-dose pulsed fluoroscopy, but the digital spot image is comparable to conventional fluoroscopy.

and its lifelong implications. ${ }^{20,23,28,30}$ It has been this increased awareness in other specialties that have a high fluoroscopy need that has driven the application of pulsed fluoroscopy and digital spot image acquisition to minimize radiation in those fields. Investigators in the disciplines of interventional cardiology, orthopedics, and urology have published widely on the modification of fluoroscopic technique to decrease radiation. ${ }^{1,6,7,10,17}$ The concern for radiation exposure to children has driven modification of fluoroscopic techniques in the realm of pediatrics. ${ }^{2,5,8,9,16,21,36}$ Collectively, these efforts have resulted in lower exposures to ionizing radiation for the surgeon and the patient. Applying these lessons learned from other fields to minimally invasive spine surgery will yield a similar result.

\section{Conclusions}

Altering the fluoroscopic technique to low-dose pulsed fluoroscopy and digital spot images can dramatically decrease fluoroscopy times and radiation doses in MIS TLIFs without compromising image quality, accuracy of pedicle screw placement, or efficiency of the procedure. The current report has demonstrated that such a protocol has the capacity to decrease the amount of fluoroscopy needed for MIS TLIFs. Over a surgeon's career, such a protocol will significantly impact the lifetime dose of radiation exposure.

\section{Disclosure}

No financial support was received for the generation of this study. No grant assistance was received for the generation of this study. The authors have no financial interest in the materials mentioned in this paper.

Author contributions to the study and manuscript preparation include the following. Conception and design: Tumialán, Clark. Acquisition of data: Tumialán, Clark, Jasmer. Analysis and interpretation of data: Tumialán, Clark. Drafting the article: Tumialán, Clark. Critically revising the article: Tumialán, Clark, Marciano. Reviewed submitted version of manuscript: Tumialán. Administrative/technical/material support: Marciano. Study supervision: Tumialán, Marciano.

\section{References}

1. Aufrichtig R, Xue P, Thomas CW, Gilmore GC, Wilson DL: Perceptual comparison of pulsed and continuous fluoroscopy. Med Phys 21:245-256, 1994

2. Belanger B, Boudry J: Management of pediatric radiation dose using GE fluoroscopic equipment. Pediatr Radiol 36 (Suppl 2):204-211, 2006

3. Bevelacqua JJ: Practical and effective ALARA. Health Phys 98 (Suppl 2):S39-S47, 2010

4. Bindal RK, Glaze S, Ognoskie M, Tunner V, Malone R, Ghosh $\mathrm{S}$ : Surgeon and patient radiation exposure in minimally invasive transforaminal lumbar interbody fusion. Clinical article. J Neurosurg Spine 9:570-573, 2008

5. Brown PH, Thomas RD, Silberberg PJ, Johnson LM: Optimization of a fluoroscope to reduce radiation exposure in pediatric imaging. Pediatr Radiol 30:229-235, 2000

TABLE 4: Fluoroscopy time in reported MIS series*

\begin{tabular}{lcccc}
\hline \multicolumn{1}{c}{ Authors \& Year } & $\begin{array}{c}\text { Mean Fluoro Time/ } \\
\text { Case }(\mathrm{sec})\end{array}$ & $\begin{array}{c}\text { Mean No. of Levels } \\
\text { Treated }\end{array}$ & $\begin{array}{c}\text { Mean Fluoro Time/ } \\
\text { Level (sec) }\end{array}$ & $\begin{array}{c}\text { Conversion Factor } \\
(0.15 \mathrm{sec})\end{array}$ \\
\hline Villavicencio et al., 2005 & 133 & 1 & 133 & 20 \\
Bindal et al., 2008 & 101.4 & 1.38 & 73.5 & 11 \\
Kim et al., 2008 & 147.2 & 1 (unilat) & 147.2 & 22 \\
Wang et al., 2010 & 84 & 1 & 84 & 13 \\
Wang et al., 2011 & 73 & 1.12 & 65 & 10 \\
Wang et al., 2012 & 46 & 1 & 46 & 7 \\
present study & 18.72 & 1 & 18.72 & NA \\
\hline
\end{tabular}

* Fluoro = fluoroscopy; NA = not applicable. 
6. Campbell RM, Strieper MJ, Frias PA, Jeager G, Balfour G, Costello L, et al: Quantifying and minimizing radiation exposure during pediatric cardiac catheterization. Pediatr Cardiol 26:29-33, 2005

7. Chida K, Fuda K, Saito H, Takai Y, Takahashi S, Yamada S, et al: Patient skin dose in cardiac interventional procedures: conventional fluoroscopy versus pulsed fluoroscopy. Catheter Cardiovasc Interv 69:115-122, 2007

8. Cohen MD: Can we use pulsed fluoroscopy to decrease the radiation dose during video fluoroscopic feeding studies in children? Clin Radiol 64:70-73, 2009

9. Cohen MD: Optimizing the use of pulsed fluoroscopy to reduce radiation exposure to children. J Am Coll Radiol 5: 205-209, 2008

10. den Boer A, de Feyter PJ, Hummel WA, Keane D, Roelandt JR: Reduction of radiation exposure while maintaining highquality fluoroscopic images during interventional cardiology using novel x-ray tube technology with extra beam filtering. Circulation 89:2710-2714, 1994

11. Elkoushy MA, Shahrour W, Andonian S: Pulsed fluoroscopy in ureteroscopy and percutaneous nephrolithotomy. Urology 79:1230-1235, 2012

12. Frush DP, Frush KS: The ALARA concept in pediatric imaging: building bridges between radiology and emergency medicine: consensus conference on imaging safety and quality for children in the emergency setting, Feb. 23-24, 2008, Orlando, FL-Executive Summary. Pediatr Radiol 38 (Suppl 4):S629-S632, 2008

13. Geijer H: Radiation dose and image quality in diagnostic radiology. Optimization of the dose-image quality relationship with clinical experience from scoliosis radiography, coronary intervention and a flat-panel digital detector. Acta Radiol Suppl 43:1-43, 2002

14. Goodman BS, Carnel CT, Mallempati S, Agarwal P: Reduction in average fluoroscopic exposure times for interventional spinal procedures through the use of pulsed and low-dose image settings. Am J Phys Med Rehabil [epub ahead of print], 2011

15. Härtl R, Lam KS, Wang J, Korge A, Kandziora F, Audigé L: Worldwide survey on the use of navigation in spine surgery. World Neurosurg 79:162-172, 2013

16. Hernandez RJ, Goodsitt MM: Reduction of radiation dose in pediatric patients using pulsed fluoroscopy. AJR Am J Roentgenol 167:1247-1253, 1996

17. Holmes DR Jr, Wondrow MA, Gray JE, Vetter RJ, Fellows JL, Julsrud PR: Effect of pulsed progressive fluoroscopy on reduction of radiation dose in the cardiac catheterization laboratory. J Am Coll Cardiol 15:159-162, 1990

18. Kim CW, Lee YP, Taylor W, Oygar A, Kim WK: Use of navigation-assisted fluoroscopy to decrease radiation exposure during minimally invasive spine surgery. Spine J 8:584-590, 2008

19. Kotre CJ, Charlton S, Robson KJ, Birch IP, Willis SP, Thornley M: Application of low dose rate pulsed fluoroscopy in cardiac pacing and electrophysiology: patient dose and image quality implications. Br J Radiol 77:597-599, 2004

20. Lee K, Lee KM, Park MS, Lee B, Kwon DG, Chung CY: Measurements of surgeons' exposure to ionizing radiation dose during intraoperative use of C-arm fluoroscopy. Spine (Phila Pa 1976) 37:1240-1244, 2012

21. Lu ZF, Nickoloff EL, Ruzal-Shapiro CB, So JC, Dutta AK: New automated fluoroscopic systems for pediatric applications. J Appl Clin Med Phys 6:88-105, 2005

22. Mariscalco MW, Yamashita T, Steinmetz MP, Krishnaney AA, Lieberman IH, Mroz TE: Radiation exposure to the surgeon during open lumbar microdiscectomy and minimally invasive microdiscectomy: a prospective, controlled trial. Spine (Phila Pa 1976) 36:255-260, 2011
23. Mulconrey DS: Fluoroscopic radiation exposure in spinal surgery: in vivo evaluation for operating room personnel. J Spinal Disord Tech [epub ahead of print], 2013

24. Nickoloff EL: AAPM/RSNA physics tutorial for residents: physics of flat-panel fluoroscopy systems: Survey of modern fluoroscopy imaging: flat-panel detectors versus image intensifiers and more. Radiographics 31:591-602, 2011

25. Payer M: "Minimally invasive" lumbar spine surgery: a critical review. Acta Neurochir (Wien) 153:1455-1459, 2011

26. Rauch P, Lin PJ, Balter S, Fukuda A, Goode A, Hartwell G, et al: Functionality and operation of fluoroscopic automatic brightness control/automatic dose rate control logic in modern cardiovascular and interventional angiography systems: a report of Task Group 125 Radiography/Fluoroscopy Subcommittee, Imaging Physics Committee, Science Council. Med Phys 39:2826-2828, 2012

27. Scanavacca M, d'Avila A, Velarde JL, Reolão JB, Sosa E: Reduction of radiation exposure time during catheter ablation with the use of pulsed fluoroscopy. Int J Cardiol 63:71-74, 1998

28. Smith DL, Heldt JP, Richards GD, Agarwal G, Brisbane WG, Chen CJ, et al: Radiation exposure during continuous and pulsed fluoroscopy. J Endourol 27:384-388, 2013

29. Smith HE, Welsch MD, Sasso RC, Vaccaro AR: Comparison of radiation exposure in lumbar pedicle screw placement with fluoroscopy vs computer-assisted image guidance with intraoperative three-dimensional imaging. J Spinal Cord Med 31:532-537, 2008

30. Ul Haque M, Shufflebarger HL, O'Brien M, Macagno A: Radiation exposure during pedicle screw placement in adolescent idiopathic scoliosis: is fluoroscopy safe? Spine (Phila Pa 1976) 31:2516-2520, 2006

31. Villavicencio AT, Burneikiene S, Bulsara KR, Thramann JJ: Utility of computerized isocentric fluoroscopy for minimally invasive spinal surgical techniques. J Spinal Disord Tech 18:369-375, 2005

32. Wang J, Zhou Y, Feng Zhang Z, Qing Li C, Jie Zheng W, Liu $\mathrm{J}$ : Comparison of clinical outcome in overweight or obese patients after minimally invasive versus open transforaminal lumbar interbody fusion. J Spinal Disord Tech [epub ahead of print], 2012

33. Wang J, Zhou Y, Zhang ZF, Li CQ, Zheng WJ, Liu J: Comparison of one-level minimally invasive and open transforaminal lumbar interbody fusion in degenerative and isthmic spondylolisthesis grades 1 and 2. Eur Spine J 19:1780-1784, 2010

34. Wang J, Zhou Y, Zhang ZF, Li CQ, Zheng WJ, Liu J: Minimally invasive or open transforaminal lumbar interbody fusion as revision surgery for patients previously treated by open discectomy and decompression of the lumbar spine. Eur Spine J 20:623-628, 2011

35. Webb J, Gottschalk L, Lee YP, Garfin S, Kim C: Surgeon perceptions of minimally invasive spine surgery. SAS J 2:145, 2008

36. Wesenberg RL, Amundson GM: Fluoroscopy in children: low-exposure technology. Radiology 153:243-247, 1984

37. Willis CE, Slovis TL: The ALARA concept in radiographic dose reduction. Radiol Technol 76:150-152, 2004

Manuscript submitted April 11, 2013.

Accepted May 14, 2013.

Please include this information when citing this paper: DOI: 10.3171/2013.5.FOCUS13144.

Address correspondence to: Luis M. Tumialán, M.D., c/o Neuroscience Publications, Barrow Neurological Institute, St. Joseph's Hospital and Medical Center, 350 W. Thomas Rd., Phoenix, AZ 85013.email: Neuropub@dignityhealth.org. 